\title{
Lusioersily
}

\section{Towards the Implementation of Rayleigh-Taylor Instability into the Multi-Phenomena Deflagration Model}

Keenan, J., Makarov, D., \& Molkov, V. (2013). Towards the Implementation of Rayleigh-Taylor Instability into the Multi-Phenomena Deflagration Model. In Unknown Host Publication (pp. 932-941). Research Publishing. https://doi.org/10.3850/978-981-07-5936-0_14-06

Link to publication record in Ulster University Research Portal

\section{Published in:}

Unknown Host Publication

Publication Status:

Published (in print/issue): 01/05/2013

DOI:

10.3850/978-981-07-5936-0_14-06

\section{Document Version}

Publisher's PDF, also known as Version of record

\section{General rights}

Copyright for the publications made accessible via Ulster University's Research Portal is retained by the author(s) and / or other copyright owners and it is a condition of accessing these publications that users recognise and abide by the legal requirements associated with these rights.

\section{Take down policy}

The Research Portal is Ulster University's institutional repository that provides access to Ulster's research outputs. Every effort has been made to ensure that content in the Research Portal does not infringe any person's rights, or applicable UK laws. If you discover content in the Research Portal that you believe breaches copyright or violates any law, please contact pure-support@ulster.ac.uk. 


\title{
Towards the Implementation of Rayleigh-Taylor Instability into the Multi-Phenomena Deflagration Model
}

\author{
Keenan, J.*, Makarov, D., and Molkov, V. \\ Hydrogen Safety Engineering and Research Centre (HySAFER), \\ University of Ulster, Newtownabbey, Co. Antrim, UK. \\ *Corresponding authoremail: j.keenan@ulster.ac.uk
}

\begin{abstract}
Data obtained from experiments carried out at the FM Global large scale deflagration chamber have been used to further develop the multi-phenomena deflagration model. This model has been under development at the University of Ulster during the last decade. This expansion of the deflagration model accounts for the inclusion of Rayleigh-Taylor (RT) instability, as an additional time-dependent combustion enhancing mechanism. The previous version of the LES deflagration model without the addition of RT instability underpredicted the experimental results, due to the model not properly capturing the external deflagration observed during the experiment. Following this underprediction, RT instability was identified as playing a significant role in combustion enhancement as the flame front accelerated towards the vent and during combustion outside the enclosure. The implementation of a mechanism to account for RT instability, in the form of an additional time-dependent flame wrinkling factor, led to an intensification of the external deflagration. This resulted in closer agreement between the simulated and experimental pressure transients.
\end{abstract}

KEYWORDS: Rayleigh-Taylor instability, vented deflagration, external deflagration, simulations.

\section{NOMENCLATURE}

\begin{tabular}{|c|c|c|c|}
\hline$A_{i, t}$ & Atwood number (-) & $\varepsilon_{t}$ & overall thermokinetic index (-) \\
\hline$A_{T}$ & turbulent surface area $\left(\mathrm{m}^{2}\right)$ & $\lambda_{i, t}$ & most unstable wavelength (m) \\
\hline$c$ & combustion progress variable (-) & $\mu$ & dynamic viscosity $(\mathrm{Pa} \cdot \mathrm{s})$ \\
\hline$D$ & flame surface fractal dimension (-) & $\mu_{e f f}$ & effective dynamic viscosity $(\mathrm{Pa} \cdot \mathrm{s})$ \\
\hline$D_{L}$ & lower limit, $D_{L}=2.05(-)$ & $v_{T, i, t}$ & turbulent kinematic viscosity $\left(\mathrm{m}^{2} / 2\right)$ \\
\hline$D_{T}$ & upper limit, $D_{L}=2.35(-)$ & $\Xi_{f}$ & factor representing fractal theory (-) \\
\hline E & expansion ratio (-) & $\Xi_{K}$ & flame self-induced turbulence factor (-) \\
\hline$g_{i, t}$ & acceleration $\left(\mathrm{m} / \mathrm{s}^{2}\right)$ & $\Xi_{K}^{\max }$ & maximum Karlovitz factor (-) \\
\hline$h_{i, t}$ & RT amplitude at current timestep (m) & $\Xi_{l p}$ & leading point factor (-) \\
\hline$h_{i, t-\Delta t}$ & RT amplitude at previous timestep $(\mathrm{m})$ & $\Xi_{l p}^{\max }$ & maximum leading point factor $(-)$ \\
\hline$k$ & wavenumber, $k=2 \cdot \pi / \lambda(1 / \mathrm{m})$ & $\Xi_{R T}$ & RT instability factor (-) \\
\hline$k_{h}$ & constant multiplier (-) & $\rho$ & density $\left(\mathrm{kg} / \mathrm{m}^{3}\right)$ \\
\hline$m_{0}$ & temperature index (-) & $\psi$ & model constant (-) \\
\hline$n_{0}$ & baric index (-) & $\omega_{i, t}$ & growth rate of perturbation $(1 / \mathrm{s})$ \\
\hline$p$ & pressure $(\mathrm{Pa})$ & Bars & \\
\hline$p_{0}$ & initial pressure $(\mathrm{Pa})$ & - & LES filtered quantity \\
\hline $\operatorname{Pr}$ & Prandtl number (-) & $\sim$ & LES mass-weighted filtered quantity \\
\hline$R$ & flame radius / integral scale $(\mathrm{m})$ & \multicolumn{2}{|c|}{ Subscripts } \\
\hline$R_{0}$ & critical radius (m) & $a$ & Air \\
\hline$R_{0, l p}$ & half of critical radius, $R_{0, l p}=R_{0} / 2(\mathrm{~m})$ & $c$ & source term, progress variable \\
\hline$S_{c}$ & progress variable source term $\left(\mathrm{kg} / \mathrm{m}^{3}-\mathrm{s}\right)$ & $E$ & source term, energy conservation \\
\hline Sc & Schmidt number (-) & eff & effective \\
\hline$S c_{e f f}$ & effective Schmidt number (-) & $h$ & amplitude \\
\hline$S_{E}$ & energy source term $\left(\mathrm{kg} / \mathrm{m}^{3}-\mathrm{s}\right)$ & $\mathrm{H}_{2}$ & hydrogen \\
\hline
\end{tabular}

Proc. of the Seventh International Seminar on Fire \& Explosion Hazards (ISFEH7), pp. 932-941

Edited by D. Bradley, G. Makhviladze, V. Molkov, P. Sunderland, and F. Tamanini

Copyright @ 2013 University of Maryland :: Published by Research Publishing

ISBN: 978-981-07-5936-0 :: doi: 10.3850/978-981-07-5936-0_14-06

www.rpsonline.com.sg 


\begin{tabular}{|c|c|c|c|}
\hline$S_{T}$ & turbulent burning velocity $(\mathrm{m} / \mathrm{s})$ & $i, j, k$ & spatial coordinate indexes \\
\hline$S_{T, i, t}$ & $S_{T}$ at current timestep $(\mathrm{m} / \mathrm{s})$ & $L$ & laminar \\
\hline$S_{u}$ & laminar burning velocity $(\mathrm{m} / \mathrm{s})$ & $\max$ & maximum \\
\hline$S_{u 0}$ & $S_{u}$ at initial conditions $(\mathrm{m} / \mathrm{s})$ & $\min$ & minimum \\
\hline$S_{u}^{w}$ & $\begin{array}{l}\text { SGS wrinkled flame burning velocity } \\
(\mathrm{m} / \mathrm{s})\end{array}$ & $T$ & turbulent \\
\hline$t$ & time $(\mathrm{s})$ & $t$ & time \\
\hline$T$ & temperature $(\mathrm{K})$ & $u$ & unburned \\
\hline$T_{u 0}$ & unburned $\mathrm{T}$ at initial conditions $(\mathrm{m} / \mathrm{s})$ & $w$ & wrinkling \\
\hline$u^{\prime}$ & sub-grid scale velocity $(\mathrm{m} / \mathrm{s})$ & $t-\Delta t$ & previous timestep \\
\hline$x_{j}$ & spatial coordinate (-) & 0 & initial conditions \\
\hline$Y_{a}$ & mass fraction of air $(-)$ & \multicolumn{2}{|c|}{ Abbreviations } \\
\hline$Y_{H_{2}}$ & mass fraction of hydrogen $(-)$ & $\mathrm{CV}$ & control volume \\
\hline Greek & & LES & Large eddy simulation \\
\hline$\alpha$ & constant coefficient (-) & RNG & renormalization group \\
\hline$\Delta t$ & timestep (s) & RT & Rayleigh-Taylor \\
\hline$\Delta t_{i g n}$ & time of ignition (s) & SGS & sub-grid scale \\
\hline$\varepsilon$ & length scale, inner cutoff (m) & UDF & user defined function \\
\hline$\varepsilon_{R_{0}} / \varepsilon$ & ratio of inner cutoff scales, $R_{0}$ and $R(-)$ & & \\
\hline
\end{tabular}

\section{INTRODUCTION}

The HySAFER Centre at the University of Ulster is working towards understanding and predicting the underlying physical phenomena of various hydrogen release and deflagration scenarios through a Large Eddy Simulation (LES) modelling approach. A description of the current multi-phenomena LES deflagration model can be found elsewhere [1] and is also briefly introduced below.

Previous work carried out at HySAFER and described in [2] investigated the cause(s) of the under prediction of maximum overpressures when this model was initially applied to experiments carried out in a mock-up hydrogen refuelling station. The outcome of this analysis identified Rayleigh-Taylor (RT) instability as the most likely missing mechanism which would, if implemented into the deflagration model, contribute to combustion enhancement in flame front areas where there was significant flow acceleration in the direction from combustion products to the fresh mixture. The primary purpose of this paper is to present an extension to the LES deflagration model, concentrating on the development of the model to take the increase in the flame front area produced by RT instability into account. This extension to the model was then tested against the experimental results published in [3].

\section{EXPERIMENT}

The experiment as described in [3] was performed at the FM Global $63.7 \mathrm{~m}^{3}(4.6 \mathrm{~m} \times 4.6 \mathrm{~m} \times 3$ $\mathrm{m}$ ) large scale test chamber, with a square vent of $5.4 \mathrm{~m}^{2}$ located on one of the vertical walls. In this experiment hydrogen concentration was $18 \%$ by volume. Ignition occurred at the centre of the chamber. Four pressure transducers were mounted to the inside of the chamber. The initial mixture was supplied by injecting the pure fuel through an inlet at floor level while mixing fans within the chamber were used to create a uniform mixture. Prior to ignition, the unburned mixture was contained within the chamber using a $0.02 \mathrm{~mm}$ thin sheet of polypropylene. Ignition was supplied using a carbon rod igniter. Pressure-time histories were provided, recorded by transducer P1 (located inside the chamber - 'Internal'). This allowed detailed comparison with simulation results permitting model analysis. 


\section{LES MODEL OVERVIEW}

The governing equations implemented are obtained by filtering the dimensional conservation equations governing mass, momentum, energy and species concentration. These equations are detailed in various publications, including [4].

\section{Model Describing Premixed Flame Propagation}

The progress variable, which is defined as the mass fraction of the products of combustion, is used to model the propagation of the flame front

$$
\frac{\partial}{\partial t}(\bar{\rho} \widetilde{c})+\frac{\partial}{\partial x_{j}}\left(\bar{\rho} \tilde{u}_{j} \widetilde{c}\right)=\frac{\partial}{\partial x_{j}}\left(\frac{\mu_{e f f}}{S c_{e f f}} \frac{\partial \widetilde{c}}{\partial x_{j}}\right)+\bar{S}_{c} .
$$

During combustion the mass burning rate is described using the gradient method [5]

$$
\overline{S_{c}}=\rho_{u} S_{T}|\operatorname{grad} \tilde{c}|,
$$

where $|\operatorname{grad} \tilde{c}|$ is the gradient of the progress variable. Using this method the integral of the source term through the numerical flame front thickness gives the same mass burning rate per unit flame surface area, $\rho_{u} S_{T}$, independent of the size of cells in the numerical front. Therefore the calculation of the turbulent burning velocity can be decoupled from the numerical mesh, for freely propagating flames. The numerical flame thickness typically spreads through 3-5 cells.

The effective viscosity is calculated according to the renormalization group (RNG) theory [6]. The RNG model of turbulence does not contain any adjustable coefficients or ad hoc parameters. During the present simulations the molecular Prandtl number and Schmidt numbers are both set to 0.7 , reflecting the characteristics for air. The effective Prandtl and Schmidt number is calculated according to RNG theory [6]. The simulations undertaken investigate a real world experimental problem, which was performed at large scales. Therefore in order to reproduce experimental flow dynamics, a combustion model accounting for key phenomena, must be introduced to the simulations. In the present study such a model has been implemented through the utilisation of an appropriate User Defined Function (UDF). This capability is available within the solver employed, ANSYS FLUENT.

\section{Multi-Phenomena Turbulent Burning Velocity Model}

The multi-phenomena deflagration model presented in this study is under continuous development, the latest version of this model is described in [1]. It is based on the progress variable equation, Eq. 1, and the gradient method, Eq. 2, for flame propagation. This version of the LES deflagration model takes into account various phenomena which are known to have a significant influence on the burning velocity: changes of pressure and temperature in the unburned gas, flow turbulence, turbulence generated by the flame front itself, $\Xi_{K}$, preferential diffusion of turbulent flames at different curvature radii (so-called leading point concept), $\Xi_{l p}$, and, when considering large scale scenarios, fractals increase of the turbulent flame surface area, $\Xi_{f}$. Following the inclusion of these different phenomena the equation for turbulent burning velocity is written as

$$
S_{T}=S_{u}^{w} \cdot \exp \left(u^{\prime} / S_{T}\right)^{2}=\left[S_{u} \cdot \Xi_{K} \cdot \Xi_{l p} \cdot \Xi_{f}\right] \cdot \exp \left(u^{\prime} / S_{T}\right)^{2},
$$

where $u^{\prime}$ is the sub-grid scale velocity [7] and $S_{T}$ is the turbulent burning velocity. Equation 3 is a modified form of Yakhot's original equation [8] for turbulent premixed flame propagation velocity. The key step in the development of this model was the substitution of the laminar burning velocity term, $S_{u}$, in Yakhot's original equation with the sub-grid scale (SGS) wrinkled 
burning velocity, $S_{u}^{w}$. This introduced term, $S_{u}^{w}$, accounts for the unresolved phenomena within the simulations affecting burning rate at all SGS lengths. It should be noted that $S_{u}^{w}$ influences the total turbulent burning rate through interaction with flow turbulence in the unburned mixture, which is accounted for within the modified form of Yakhot's equation, Eq. 3. Each of the combustion phenomena included in this version of the model [1] will now be briefly summarised.

\section{Changes of concentration, pressure and temperature}

The variation in the value of laminar burning velocity throughout the flammable concentration range of hydrogen, $Y_{H_{2}}$, is adopted based on [9]. The effect of concentration is taken into account through the value of the initial burning velocity, $S_{u 0}$. Additionally the dependence of the laminar burning velocity on transient pressure, $p$, and temperature, $T$, is taken into account within the model following the assumption of adiabatic compression / expansion

$S_{u}\left(Y_{H_{2}}, T, p\right)=S_{u 0}\left(Y_{H_{2}}\right) \cdot\left(\frac{T}{T_{u 0}}\right)^{m_{0}\left(Y_{H_{2}}\right)}\left(\frac{p}{p_{0}}\right)^{n_{0}\left(Y_{H_{2}}\right)}=S_{u 0}\left(T_{H_{2}}\right) \cdot\left(\frac{p}{p_{0}}\right)^{\varepsilon_{t}\left(T_{H_{2}}\right)}$,

where the thermokinetic index can be calculated as $\varepsilon_{t}=m_{0}+n_{0}-m_{0} / \gamma$ and $\gamma$ is the adiabatic index (specific heat ratio) of the unburned mixture. The values for the overall and thermal thermokinetic indices are taken from [10], as $m_{0}=2.3$ and $\varepsilon=0.65$.

\section{Turbulence generated by the flame front itself}

The flame front constitutes a flow source that leads to the introduction of additional turbulence into the flow by combustion, the maximum intensity of which can be derived from [11]. The upper limit for the flame wrinkling factor associated with flame generated turbulence may be written as [12]: $\Xi_{K}^{\max }=(E-1) / \sqrt{3}$. The implemented mathematical model for this mechanism takes into account the critical radius for the transition from a laminar to a fully turbulent flame

$$
\Xi_{K}=1+\left(\psi \cdot\left[\Xi_{K}^{\max }-1\right]\right) \cdot\left(1-\exp \left[-R / R_{0}\right]\right) \text {, }
$$

where $R$ is the distance from the point of ignition to the flame front, $R_{0}$ is the characteristic radius at which transition to the fully turbulent self-similar regime occurs, and $\psi$ is an 'ad-hoc' parameter contained within the model, $\psi \leq 1$. This wrinkling factor increases from a value of 1.0 at the point of ignition (in an initially quiescent mixture) up to $\Xi_{K}^{\max }$ for a fully developed turbulent flame. Following [13] the value of $R_{0}=1.0 \mathrm{~m}$ is used during simulations. The term, $\psi$, can be considered to represent the extent to which the theoretical maximum of this mechanism can be reached. The value $\psi=0.5$ is adopted in the present study following [14].

\section{Preferential diffusion and flame stretch}

As confirmed experimentally in [15] due to thermo-diffusive instability the hydrogen flame will propagate with a cellular structure, even in the absence of turbulence, and for a particular mixture composition there exists a curvature radius which will produce a maximum mass burning rate. As argued in [16] the turbulent burning velocity is controlled by the flamelets, with curvature, that advance most into the unburned mixture. According to this concept the hydrogen concentration at the leading points were determined and corresponding values of burning velocities were calculated using linear interpolation [17]. Within the multi-phenomena deflagration model it has been assumed that the described preferential-diffusive instability develops linearly with the flame size and is then set to reach its maximum value at half of the critical radius, $R_{0, l p}=R_{0} / 2$, after this point it is then set to remain constant, $\Xi_{l p}^{\max }$, 
$\Xi_{l p}=\operatorname{MIN}\left[\Xi_{l p}^{\max } ; 1+\frac{\left(\Xi_{l p}^{\max }-1\right) \cdot R}{R_{0, l p}}\right]$

\section{Fractal increase of the turbulent flame surface area}

The mathematics of fractals has been primarily developed by Mandelbrot [18]. Following this work, as outlined in [19], the flame surface area of an outwardly propagating turbulent flame will grow faster than a spherical laminar flame. The equation describing this growth can be written as: $A_{T} \approx \varepsilon^{2-D} R^{D}$, where $A_{T}$ is turbulent surface area, $\varepsilon$ is one length scale (inner cut-off), $R$ is another length scale (outer cut-off) and $D$ is the fractal dimension. The fractal dimension, $D$, "is a measure of the degree of wrinkling and fragmentation of the flame front" [20]. The equation describing the change in the value of $D$ with laminar burning velocity and root mean square velocity was formalised in [21]. When implementing fractal theory into Ulster's deflagration model, it is considered that the integral scale of the problem forms the outer cut-off. Additionally, as suggested in [22], the inner cut-off is assumed to be proportional to the laminar flame thickness. This mechanism is set to take effect starting from the critical radius, $R_{0}=1.0 \mathrm{~m}$, so that it is applied only to a fully developed turbulent flame

$$
\Xi_{f}=\operatorname{MAX}\left[1 ;\left(\frac{R}{R_{0}} \cdot \frac{\varepsilon_{R_{0}}}{\varepsilon}\right)^{D-2}\right] \text {. }
$$

This model, Eq. 3, was then used to simulate the experiment performed by FM Global [3].

\section{Numerical Details}

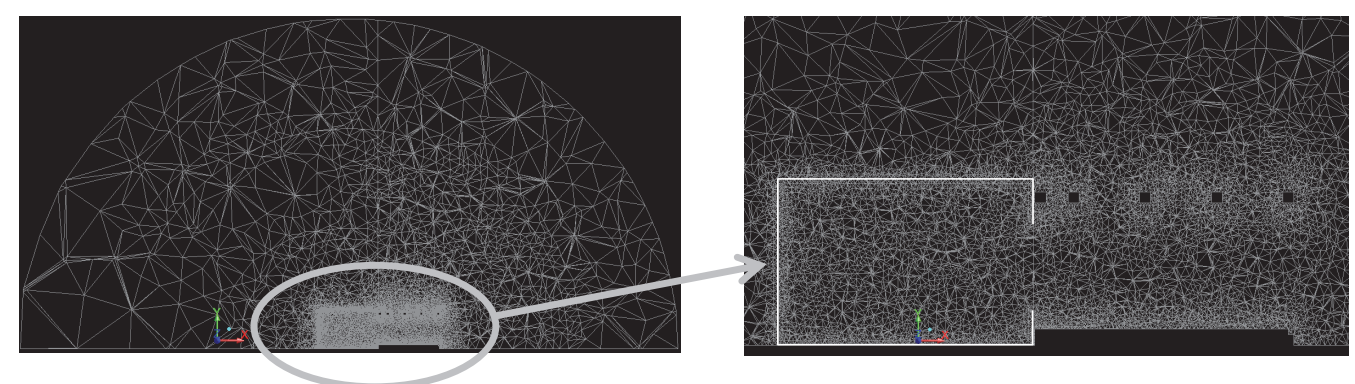

Figure 1. Calculation domain and mesh: overall view of domain (left), enlarged chamber view (right).

The calculation domain as shown in Fig. 1 comprises a large hemispherical area of radius $25 \mathrm{~m}$. At its centre is a representation of the FM Global large scale deflagration facility described in [3]. This large area was created in order to exclude the effects of boundary conditions on the external deflagration and to accommodate the diverging pressure wave generated. The calculation domain was meshed using an unstructured tetrahedral grid. During this analysis the smallest control volumes (CVs) were located inside the chamber and in the area immediately outside the vent, where the external deflagration takes place. The average edge size of the CVs located inside the chamber and around the vent was $0.1 \mathrm{~m}$, this clearly implies SGS modelling of unresolved combustion mechanisms as mentioned above. The characteristic CV size was then smoothly increased within the rest of the calculation domain. The total number of CVs was 989,339. Simulations were performed on the CFD platform ANSYS FLUENT (release 13.0). As a tetrahedral mesh was implemented, in order to obtain more accurate results, the governing equations were solved by employing a second-order accurate upwind scheme for convection terms. Additionally from authors' experience the reduction of discretization scheme order from $2^{\text {nd }}$ to $1^{\text {st }}$ resulted in a significant decrease of combustion rate and underestimation of pressure peaks. The diffusion terms were central-differenced and second-order accurate. The progress 
variable and energy source terms were solved within the UDF capability available when using ANSYS FLUENT. An explicit scheme was used for time stepping, where the CourantFriedrichs-Lewy (CFL) number was set to 0.8 to ensure stability. This resulted in a time step of the order of $10^{-6} \mathrm{~s}$.

The boundary conditions applied were non-slip, non-permeable, adiabatic conditions on all walls and ground surfaces, not including the chamber walls. At the outer edge of the calculation domain the non-reflecting pressure far-field boundary condition, as implemented by ANSYS FLUENT based on Riemann invariants, was applied. An unrestricted open vent was used in all simulations. At initial conditions the flammable mixture was contained inside the chamber and air was located in the remaining area of the calculation domain. The pressure was set to atmospheric and initial temperature was $295 \mathrm{~K}$. Inside the calculation domain the initial value of the progress variable was set to $c=0$. Inside the chamber the initial mass fraction of fuel, hydrogen, was $Y_{H_{2}}=0.015$ (corresponding to $18 \%$ of hydrogen by volume) and the mass fraction of air was $Y_{a}=0.985$. Combustion was initiated by slowly increasing the progress variable in one $\mathrm{CV}$. The duration of ignition was assumed to be equal to the flame propagation time from the centre to the edge of the ignition CV: $\Delta t_{i g n}=1 / 2 \cdot\left(\Delta_{c v} / S_{u} \cdot E\right)$, where $\Delta_{c v}$ is the ignition $\mathrm{CV}$ size (equivalent radius).

\section{Model Results Before Inclusion of Rayleigh-Taylor Instability Mechanism}

The simulation results shown in Fig. 2 (left) were obtained using the multi-phenomena turbulent burning velocity model described by Eq. 3, termed as the 'former' model results. Also plotted on Fig. 2 (left), for comparison purposes, are the pressure dynamics obtained from the experiment [3]. As shown in Fig. 2 (left) the former model failed to satisfactorily reproduce the experimental pressure dynamics, most notably the first distinct pressure peak. As described in [23] (and following the general description contained in [24]), this pressure peak can be said to be caused by the external deflagration, which is often called "external explosion".

In order for this significant first pressure peak to occur the pressure generated by the external explosion must be at least equal to or above the internal pressure. Such a pressure increase externally will reduce the pressure difference across the vent thereby reducing the venting efficiency for the duration of the external explosion [1],[25]. This blocking of the outflow from the chamber will cause the internal pressure to increase, until the external explosion has dissipated. External pressure dissipation will release the pressure inside and cause the production of the pressure peak generated during the experiment. As shown in Fig. 3 (left) the external pressure obtained from the former model is not of sufficient strength to have a significant influence on the internal pressure dynamics. This underprediction can be attributed to a combustion enhancing mechanism not being accounted for within the former model.

\section{MODELLING OF RAYLEIGH-TAYLOR INSTABILITY}

Rayleigh-Taylor (RT) instability has been reported by a number of authors to occur during vented deflagration scenarios. The growth of this instability has been identified on the flame as it accelerated through the vent [26], close to the vent after the hot combustion gases leave the chamber [27] and also inside the chamber [24], [27] and [28]. Additionally, during work carried out at Ulster [2], RT instability was identified as being the most likely missing contribution to the combustion enhancement in the model compared to the experiment. Such work has provided evidence for the significant influence of RT instability during vented deflagration scenarios. The mathematical model describing RT instability is summarised below. 


\section{Description of Time-Dependent RT Instability Model}

The approach undertaken to model this combustion enhancing mechanism involves the introduction of an additional transport equation to solve for the RT factor, $\Xi_{R T}$

$$
\frac{\partial \Xi_{R T}}{\partial t}+\left(U_{i}+S_{t, i}\right) \frac{\partial \Xi_{R T}}{\partial x_{i}}=S_{\Xi_{R T}},
$$

where the first term in Eq. 8 is the unsteady term, the second term is the convection term and the third term is the source term. Equation 8 is similar to the equation described in [29]. This term is then combined with the mechanisms contained in the LES deflagration model

$$
S_{T}=\left[S_{u} \cdot \Xi_{K} \cdot \Xi_{l p} \cdot \Xi_{f} \cdot \Xi_{R T}\right] \cdot \exp \left(u^{\prime} / S_{T}\right)^{2} .
$$

The source term within the developed RT transport equation accounts for the increase and reduction of flame surface area due to RT instability in combustion conditions, $S_{\Xi_{R T}}=d \Xi_{R T} / d t$. Following the experimental observations contained in [26] it has been assumed that the flame front takes on a needle-like structure when RT instability becomes dominant. Within this model $\Xi_{R T}$ takes the form of the ratio between the surface area of the perturbed and unperturbed (flat) flame front. The growth of the amplitude of the perturbation is described as $h_{i, t}=h_{i, t-\Delta t} \cdot e^{\omega t}$ [30]. The growth rate itself is controlled by acceleration, with amplitude increasing if acceleration is in the unstable direction. The stabilisation of the mechanism, the 'sink', is controlled by annihilation of the flame surface at cusps [25] and also by a reduction in amplitude if the flame accelerates in the stable direction. Following this description of the growth and removal rates associated with the developed RT instability model, the equation describing the associated RT amplitude, $h_{i, t}$, can be written as

$$
h_{i, t}=h_{i, t-\Delta t}\left(1+\omega_{i, t} \cdot \Delta t\right)-\alpha \cdot S_{T, i, t}\left(\Xi_{R T}-1\right) \Delta t,
$$

where $h_{i, t-\Delta t}$ is the amplitude of the perturbation at the previous timestep, $\omega_{i, t}$ is the growth rate and $\alpha$ is an empirical coefficient of the order of 1.0 associated with the removal term. Wavelength and subsequently growth rate are calculated as a function of acceleration. Therefore for a particular value of acceleration there is a specific corresponding most unstable wavelength [31]. The equation describing the calculation of wavelength has been obtained from [31]: $\lambda_{i, t}=4 \cdot \pi \cdot\left(v_{T, i, t}^{2} / g_{i, t} \cdot A_{i, t}\right)^{1 / 3}$, where $v_{T, i, t}$ is turbulent kinematic viscosity, $A_{i, t}$ is Atwood number and $g_{i, t}$ is acceleration. Growth rate can be described using the classical equation outlined by many authors, including [32]. Following substitution of the equation describing wavelength into the growth rate equation obtained in [32], growth rate can be written as

$$
\omega_{i, t}=\sqrt{\left(A_{i, t} \cdot g_{i, t}\right)^{4 / 3} /\left(2 \cdot\left(v_{T, i, t}\right)^{2 / 3}\right)} \text {. }
$$

To take account of the variation in acceleration, the amplitude at each timestep is re-scaled according to the value of the wavelength at the current and previous timestep, prior to the calculation of the updated amplitude. Additionally, Eq. 10 contains two parameters which must be defined within the RT model. These parameters are a multiplier required for the calculation of the initial amplitude and also $\alpha$, which is a constant term contained within the 'sink' term. The initial amplitude is calculated as a percentage of the wavelength as outlined in [33], i.e. $h_{i, t}=$ $k_{h} \cdot \lambda_{i, t}$. In the key area of expected growth in RT as outlined in [24], [27] and [28] within this parametric study, $k_{h}$ is set to 0.5 . This value for $k_{h}$ was obtained from [33]. Conversely, in this initial study, to limit the growth of $\Xi_{R T}$ in all other areas $k_{h}$ is set to 0.001 . Subsequently, within the flame front, amplitude is calculated according to Eq. 10. Following analysis of this procedure 
significant growth in the value of $\Xi_{R T}$ was reliant on and only encountered in areas where there was a sufficiently high concentration of acceleration in the unstable direction. $\alpha$ is an empirical coefficient, of the order of 1 , associated with the removal term and is set to 0.75 .

\section{Simulation Results with Inclusion of the RT Instability Model}

Figure 2 (left) shows a comparison between the experimental results and the results obtained from the former version of the multi-phenomena turbulent burning velocity model, Eq. 3 . Subsequently Fig. 2 (right) shows a comparison between the experimental results and the results obtained from the updated model, following the inclusion of the RT instability mechanism, Eq. 9.

\section{Internal pressure dynamics}
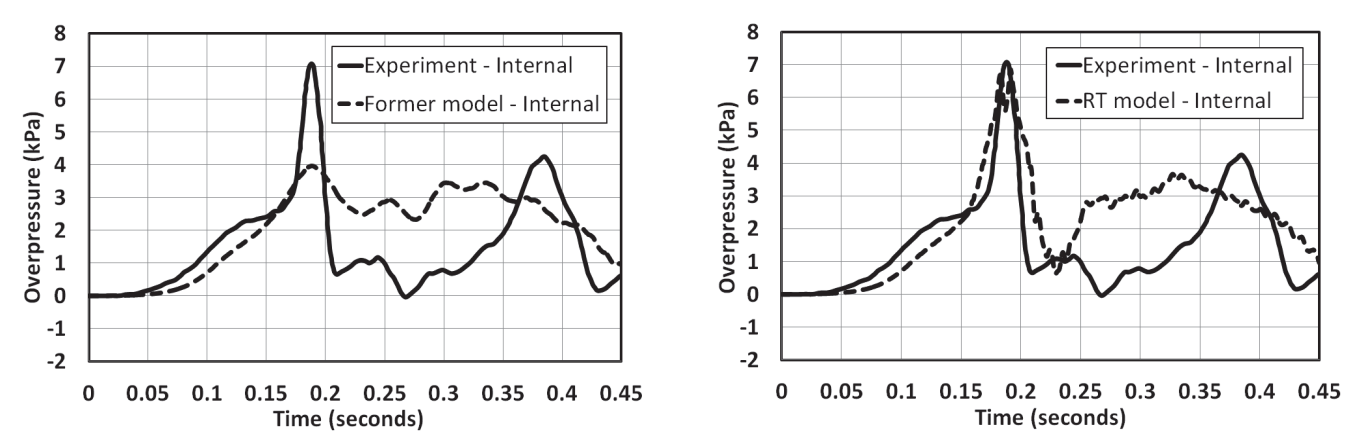

Figure 2. Comparison between experiment and former model simulation (left); and comparison between experiment and RT model (right). All pressures measured internally at location P1.

From the results presented in Fig. 2 (right), following the introduction of RT instability, the sharp pressure increase associated with the external explosion has been more closely replicated. Additionally the decrease in pressure to near atmospheric levels, following this peak, has also been reproduced. In order to produce this pressure peak the inclusion of RT instability has a significant effect on combustion enhancement outside the chamber.

External pressure dynamics
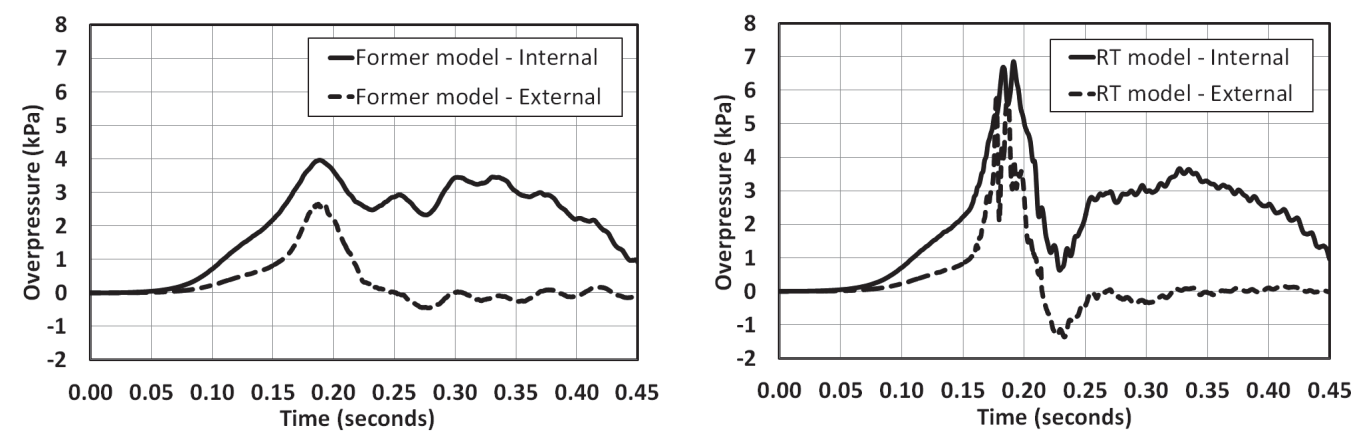

Figure 3. Former model simulation (left); and RT model simulation (right), in both figures internal pressure measured at location $\mathrm{P} 1$ and external pressure measured $1.17 \mathrm{~m}$ from vent centre.

The external pressure curves presented in Fig. 3 were recorded, during the simulation, at a distance of $1.17 \mathrm{~m}$ from the centre of the vent. From Fig. 3 (right) the introduction of RT instability has increased the pressure in the area in front of the vent, causing a reduction in the efficiency of the venting process. This intensification of the external explosion, facilitated by the 
inclusion of RT instability, created the sharp internal pressure peak recorded during the simulation, Fig. 2 (right).

As shown in Fig. 3 (left) without the inclusion of RT instability these high external pressures are not reproduced. In Fig. 3 (right) following the inclusion of RT instability the external pressure has been greatly increased. As previously stated it is expected that RT instability should arise as the flame front accelerates through the vent [26] and also close to the vent after the flame has exited the chamber [27]. As the flame exits the vent it pushes the heavier, slower moving unburned mixture. This acceleration of the colder unburned hydrogen/air mixture outside the chamber is in the unstable direction and leads to the observed increase in $\Xi_{R T}$. This increase in $\Xi_{R T}$ outside the chamber leads to an increase in turbulence and ultimately the creation of the sharp pressure peaks observed outside the chamber.

\section{CONCLUSIONS}

Rayleigh-Taylor (RT) instability was identified as having a major influence on pressure generation during the selected vented deflagration scenario. The LES deflagration model has been developed to account for this additional combustion enhancing mechanism. This updated model was then tested against the experimental data obtained from large scale experiments carried out by FM Global on the vented deflagration of a lean, 18\% hydrogen-air mixture.

The RT instability model is implemented in the form of an additional flame wrinkling factor in the multi-phenomena deflagration model, the development of which is governed by a separate transport equation, with corresponding source and sink terms based on phenomenological considerations. The implementation of this factor led to an intensification of the external deflagration as the flame reached and propagated through the vent. This high pressure external explosion had a significant influence on the internal pressure dynamics. The increase in pressure in the area surrounding the vent caused a reduction in the efficiency of the venting process, leading to an increase in the internal pressure. Following the dissipation of the external explosion these high pressure combustion products were then able to flow out of the chamber. This process, as observed experimentally, resulted in a sharp pressure peak inside the chamber.

The introduction of RT instability into the Ulster LES deflagration model led to closer agreement between the simulation and experimental results, for the considered experiment. Therefore when considering vented deflagration scenarios RT instability must be considered and implemented within sub-grid scale premixed combustion models that are designed to capture the main features and pressure transients associated with external explosions.

\section{REFERENCES}

1. Molkov, V., Fundamentals of Hydrogen Safety Engineering, parts I \& II. Free download e-book, bookboon.com, ISBN: 978-87-403-0279-0, 2012.

2. Makarov, D., Verbecke, F., Molkov, V., and Keenan, J., "On Unresolved Mechanisms of Large Scale Deflagrations in Complex Geometries," in Proceedings of the 6th ISFEH, 2010, pp. 93-103.

3. Bauwens, C.R., Chaffee, J., and Dorofeev, S.B., "Vented Explosion Overpressures from Combustion of Hydrogen and Hydrocarbon Mixtures," IJHE, vol. 36, no. 3, pp. 2329-2336, Feb. 2011.

4. Molkov, V., Makarov, D., and Schneider, H., "LES Modelling of an Unconfined Large-Scale HydrogenAir Deflagration," Journal of Physics D (Applied Physics), vol. 39, no. 20, pp. 4366-76, Oct. 2006.

5. Prudnikov, A., Combustion of Homogeneous Fuel-Air Mixtures in Turbulent Flows., Physical Principles of the Working Process in Combustion Chambers of Jet Engines, pp. 244-336. CFSTI, 1967.

6. Yakhot V., and Orszag, S.A., "Renormalization Group Analysis of Turbulence. I. Basic Theory," Journal of Scientific Computing, vol. 1, no. 1, pp. 3-51, 1986.

7. Pope, S.B., Turbulent Flows. Cambridge University Press, 2000. 
8. Yakhot, V., "Propagation Velocity of Premixed Turbulent Flames," Combustion Science and Technology, vol. 60, pp. 191-214, 1988.

9. Lamoureux, N., Djebaili-Chaumeix, N., and Paillard, C.E., "Laminar Flame Velocity Determination for H2-Air-Steam Mixtures Using The Spherical Bomb Method,” 2002, vol. 12, pp. Pr7/445-Pr7/452.

10. Babkin, S.B., Private communication. Institute of Chemical Kinetics and Combustion, Siberian Branch, Russian Academy of Science, Novosibirsk, Russia, 2003.

11. Karlovitz, B., Denniston, D.W., and Wells, F.E., "Investigation of Turbulent Flames," The Journal of Chemical Physics, vol. 19, no. 5, pp. 541- 547, 1951.

12. Molkov, V., Baratov, A.N., and Korolchenko, A.Y., "Dynamics of Gas Explosions in Vented Vessels: A Critical Review and Progress," Progress in Astronautics \& Aeronautics, vol. 154, pp. 117-131, 1993.

13. Gostintsev, Y.A., Istratov, A.G., and Shulenin, Y.V., "Self-Similar Propagation of a Free Turbulent Flame in Mixed Gas Mixtures," Combustion, Explosion and Shock Waves, vol. 24 (5), 1989.

14. Verbecke, F., "Formation and Combustion of Non-Uniform Hydrogen-Air Mixtures," Ph.D Thesis, HySAFER centre, University of Ulster, Newtownabbey, N. Ireland, U.K., 2009.

15. Bell, J.B., Cheng, R.K., Day, M.S., Beckner, V.E., and Lijewski, M.J., "Interaction of Turbulence and Chemistry in a Low-Swirl Burner," JPCS, vol. 125, no. 012027, pp. 1-5, 2008.

16. Kuznetsov, V.R. and Sabel'nikov, V.A., Turbulence and Combustion. Moscow, Russia: Hemisphere Publishing Corporation, 1990.

17. Zimont, V.L. and Lipatnikov, A.N., "A Numerical Model of Premixed Turbulent Combustion of Gases," Chemical Physics Reports, vol. 14, no. 7, p. 993, 1995.

18. Mandelbrot, B.B., The Fractal Geometry of Nature, Eighteenth printing, 1999. New York, USA: W. H. Freeman \& Co Ltd, 1983.

19. Gouldin, F.C., "An Application of Fractals to Modeling Premixed Turbulent Flames," Combustion and Flame, vol. 68, pp. 249-266, 1987.

20. Mantzaras, J., "Geometrical Properties of Turbulent Premixed Flames. Comparison Between Computed and Measured Quantities," Combustion science and technology, vol. 86, no. 1-6, pp. 135-162, 1992.

21. North, G.L. and Santavicca, D.A., "The Fractal Nature of Premixed Turbulent Flames," Combustion Science and Technology, vol. 72, no. 4-6, pp. 215-232, 1990.

22. Fureby, C., "A Fractal Flame-Wrinkling Large Eddy Simulation Model for Premixed Turbulent Combustion," Proceedings of the Combustion Institute, vol. 30, pp. 593-601, 2004.

23. Bauwens, C.R., Chaffee, J., and Dorofeev, S.B., "Experimental and Numerical Study of Hydrogen-Air Deflagrations in a Vented Enclosure," in 7th ISHPMIE proceedings Vol. 1, St. Petersburg, Russia, 2008.

24. Cooper, M.G., Fairweather, M., and Tite, J.P., "On the Mechanisms of Pressure Generation in Vented Explosions," Combustion and Flame, vol. 65, no. 1, pp. 1-14, 1986.

25. Bauwens, C.R. and Dorofeev, S.B., "Effect of the External Explosion on Vented Deflagrations," presented at the 8th ISHPMIE, Yokohama, Japan, 2010.

26. Tsuruda, T. and Hirano, T., "Growth of Flame Front Turbulence during Flame Propagation across an Obstacle," Combustion Science and Technology, vol. 51, no. 4-6, pp. 323-328, 1987.

27. Solberg, D.M., Pappas, J.A., and Skramstad, E., "Observations of Flame Instabilities in Large Scale Vent Gas Explosions," Symposium (International) on Combustion, pp. 1607-1614, 1981.

28. McCann, D.P.J., Thomas, G.O., and Edwards, D.H., "Gasdynamics of Vented Explosions. I. Experimental Studies," Combustion and Flame, vol. 59, no. 3, pp. 233-50, Mar. 1985.

29. Weller, H.G., Tabor, G., Gosman, A.D., and Fureby, C., "Application of a Flame-Wrinkling LES Combustion Model to a Turbulent Mixing Layer,” Sym. (Int.) on Combustion, vol. 1, pp. 899-907, 1998.

30. Zeldovich, Y.B., Barenblatt, G.I., Librovich, V.B., and Makhviladze, G.M., "Mathematical Theory of Combustion and Explosions," New York, NY, USA: Consultants Bur, 1985.

31. Youngs, D.L., "Numerical Simulation of Turbulent Mixing by Rayleigh-Taylor Instability," presented at the Physica D, Netherlands, 1984, vol. 12D, pp. 32-44.

32. Dimonte, G., Ramaprabhu, P., Youngs, D.L., Andrews, M.J., and Rosner, R., "Recent Advances in the Turbulent Rayleigh-Taylor Instability," Physics of Plasmas, vol. 12, no. 056301, pp. 1-6, 2005.

33. Tryggvason, G., "Numerical Simulations of the Rayleigh-Taylor Instability," Journal of Computational Physics, vol. 75, no. 2, pp. 253-82, Apr. 1988. 